\title{
Stability of a Nonlinear Quarter-Car System with Multiple Time-Delays
}

\author{
Raghavendra D. Naik and Pravin M. Singru \\ Department of Mechanical Engineering, Birla Institute of Technology and Science, Pilani, K. K. Birla Goa Cam- \\ pus, Zuarinagar, Goa, India 403726
}

(Received 20 March 2013; accepted 4 March 2015)

This paper examines the dynamical behaviour of a nonlinear oscillator which models a two-degree-of-freedom quarter-car system forced by the road profile. The influence of two time delays in the system, which is generally due to the inherent dynamics of the actuator, is studied. The asymptotic and technical stability domain is obtained by using Bogusz's stability criterion for a two-degree-of-freedom system. The results obtained from Lyapunov's and Bogusz's stability criterions are compared. The numerical results obtained are found to be in good agreement with the analytical predictions.

\section{INTRODUCTION}

Li et al. investigated a possible chaotic motion in a nonlinear vehicle suspension system, which is subject to a multifrequency excitation from a road surface. ${ }^{5}$ Litak et al. investigated global homoclinic bifurcation and the transition to chaos in the case of a quarter-car model excited kinematically by a road surface profile. ${ }^{6}$ Siewe Siewe investigated resonance, stability, and the chaotic motion of a quarter-car model excited by a road surface profile. ${ }^{11}$ All controllers exhibit a certain timedelay during operation. Many researchers have studied the behaviour of delayed differential equations. Zhang et al. studied the stability of the delayed differential equations. ${ }^{13}$ Wirkus and Rand investigated the dynamics of two van der Pol oscillators with delay velocity coupling with special attention to the bifurcation accompanying the change in number and the stability of the solutions. ${ }^{9}$ Gohary studied the vibration suppression of a dynamical system to multi-parametric excitations via a time-delay absorber. ${ }^{3}$ Naik \& Singru studied the stability of a single-degree-of-freedom system. ${ }^{8}$

The dynamics of a quarter-car model with nonlinear suspension characteristics was studied by $\mathrm{Li}$ et al. and Shen et al. ${ }^{4,10}$ Recent efforts by Borowiec et al. have been focused on the excitation of the automobile by a road surface profile with harmful noise components. ${ }^{2}$ Hysteretic nonlinear suspension was studied by Yang et al. ${ }^{12}$

This paper deals with the dynamics of a quarter-car model with an active vehicle suspension system. The influence of delay in the system, which is generally due to the inherent dynamics of the actuator, is studied. The time delay systems are usually of infinite dimension; an attempt is made to reduce them to a finite dimension since the operating time delays in the system under consideration are small (Processing Time \& Actuator Delays). The technical stability domain is obtained by using Boguszs stability criterion on the finite time domain. The asymptotic stability is derived for unequal time delays.

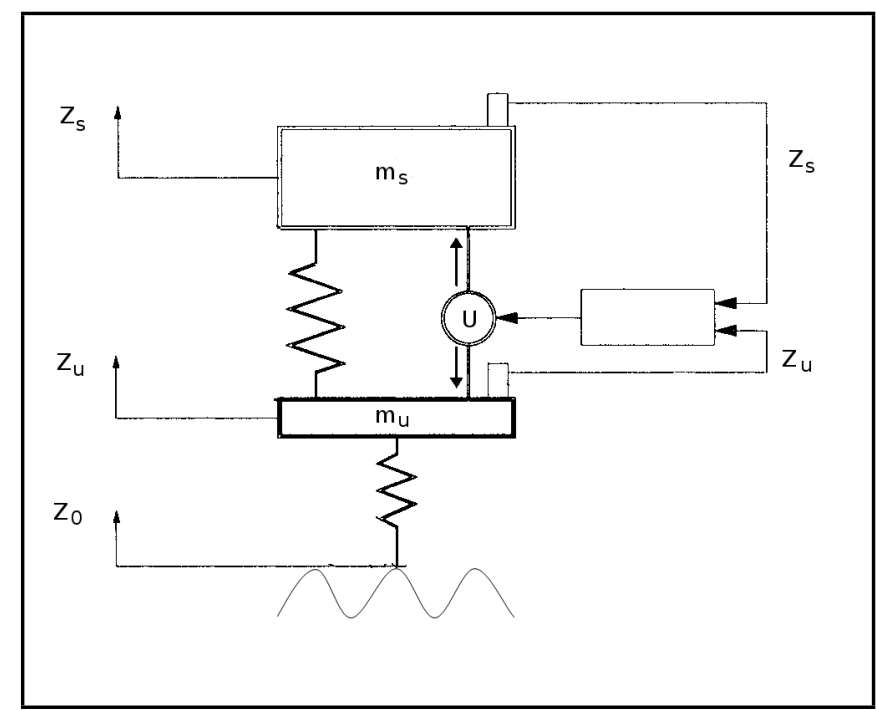

Figure 1. The quarter-car model active control.

The stability of the two models are investigated, and the results are compared. The results are validated by numerical simulations.

\section{TWO-DEGREE-OF-FREEDOM QUARTER- CAR SYSTEM}

Figure 1 shows the closed-loop active control for the vehicle system. This system represents an active vehicle suspension system with non-linearity in the dampers; the parameters considered are similar to that of the single-degree-of-freedom system considered in the previous section.

The equation of motion is assumed to have the following form:

$$
\begin{aligned}
m_{u} \ddot{Z}_{u}-k\left(Z_{s}-Z_{u}\right)-k_{t}\left(Z_{0}-Z_{u}\right)+U(t) & =0 ; \\
m_{s} \ddot{Z}_{s}+k\left(Z_{s}-Z_{u}\right)-U(t) & =0 ;
\end{aligned}
$$

